\title{
Case report 427
}

Mark Burnstein, M.D. ${ }^{1}$, Kenneth A. Buckwalter, M.D. ${ }^{1}$, William Martel, M.D. ${ }^{1}$, Kenneth D. McClatchey, M.D. ${ }^{2}$, and Douglas Quint, M.D. ${ }^{1}$

Departments of ${ }^{1}$ Radiology and ${ }^{2}$ Pathology, University of Michigan Hospitals, Ann Arbor, Michigan, USA

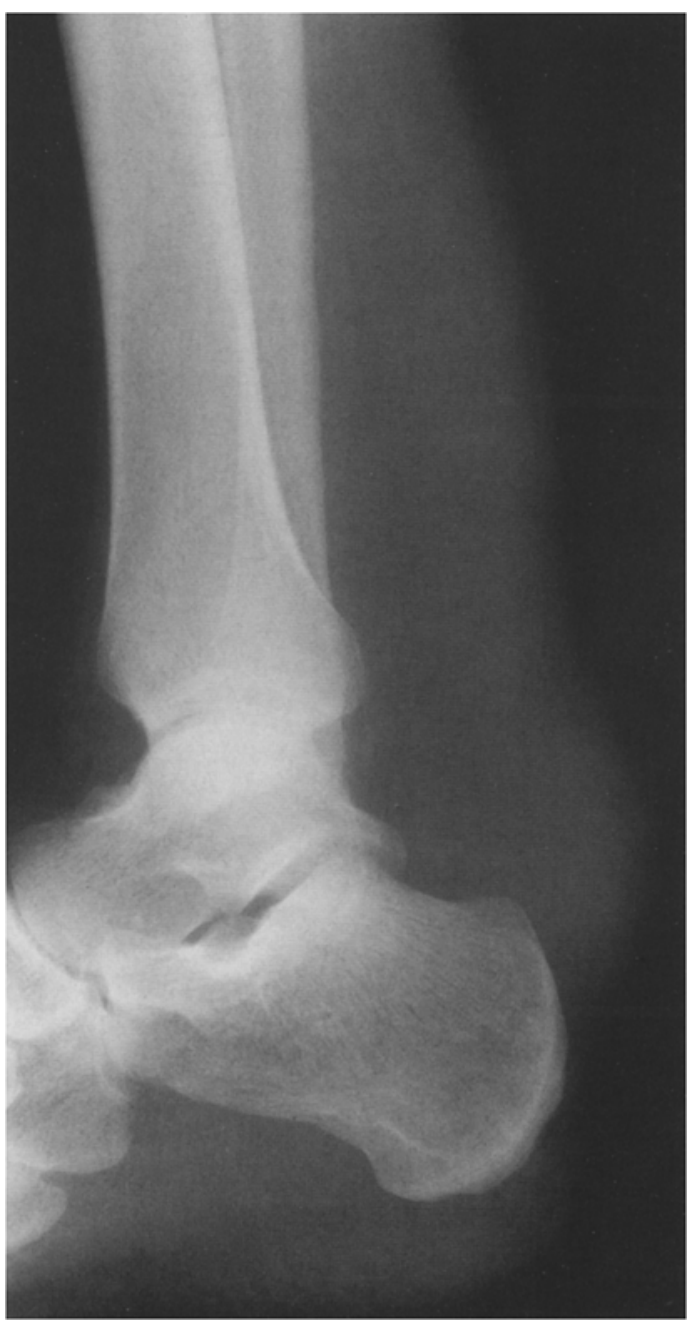

\section{Imaging studies}

Fig. 1. A lateral roentgenogram of the right ankle shows marked enlargement of the Achilles tendon

\section{Clinical information}

This 42-year-old man with a 10 year history of psychiatric disorders presented with acute changes in his mental status. Physical examination demonstrated masses over the Achilles tendons, the left knee and on the eyelids. Laboratory data demonstrated a normal serum cholesterol, normal serum uric acid, elevated serum triglycerides, and elevated cholestanol levels in the serum und urine. EEG findings were consistent with a metabolic encephalopathy. CT and MR studies were performed after a plain film study of the right ankle (Figs. 1-5).

A biopsy of a tendon was performed.

Address reprint requests to: Mark Burnstein, M.D. University of Michigan Hospitals, Department of Radiology, Taubman Center 2910-0326, 1500 E. Medical Center Drive, Ann Arbor, MI 48109-0326, USA 

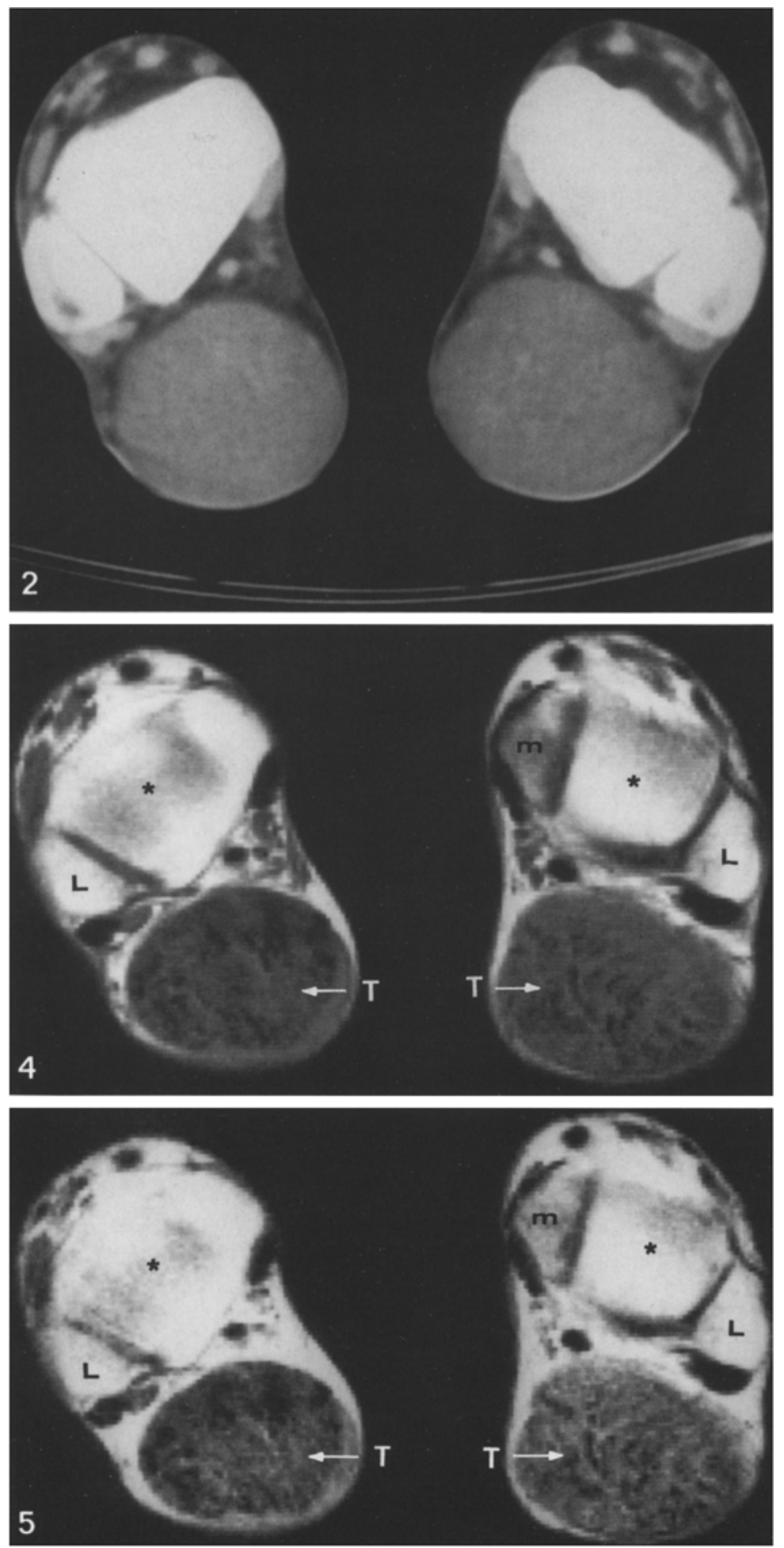

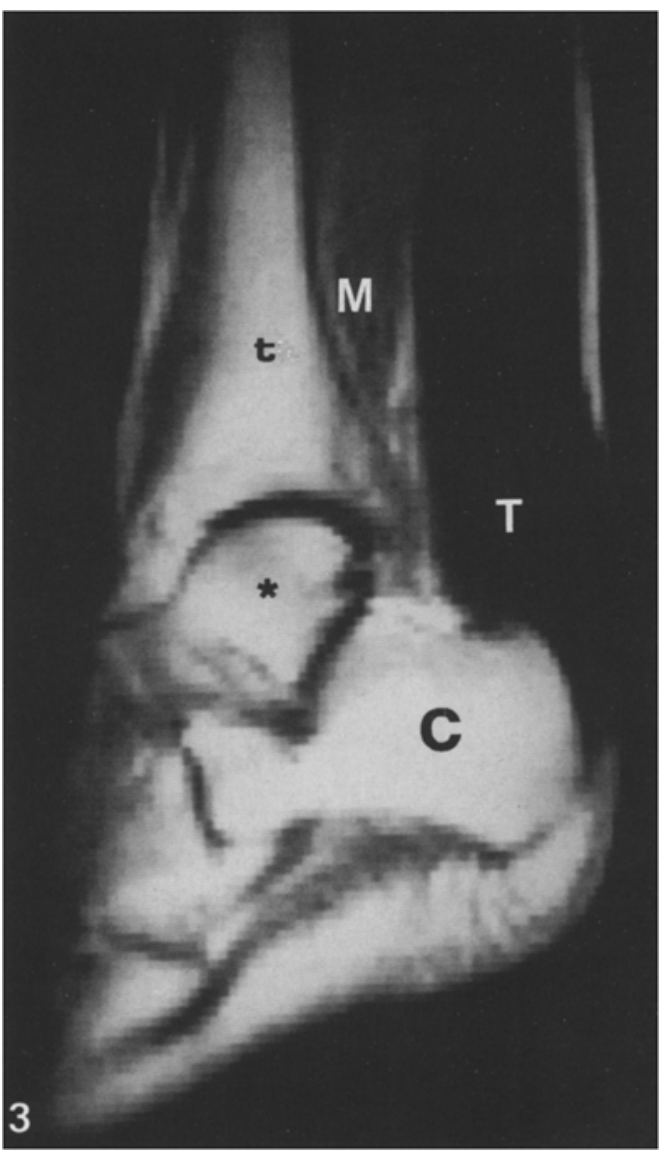

Fig. 2. An axial CT image at the level of the ankle joints demonstrates massive enlargement of the Achilles tendons, which are mildly inhomogeneous in attenuation. No calcifications are identified

Fig. 3. Magnetic resonance studies using a sagittal T1 weighted (TR $0.3 \mathrm{~s}$ TE $30 \mathrm{~ms}$ ) MR image, demonstrates the markedly enlarged Achilles tendon $(T)$. The tendon is less intense than adjacent muscle $(\mathrm{M})$

Fig. 4. An axial T1 weighted (TR $0.5 \mathrm{~s}$ TE $30 \mathrm{~ms}$ ) MR image, at the same level as previous images, shows a markedly enlarged tendon which is heterogeneous in signal intensity. $(\mathrm{L}=$ lateral malleolus; $\mathrm{m}=$ medial malleolus; $\mathrm{T}=$ talus)

Fig. 5. Axial T2 weighted (TR $1.5 \mathrm{~s}$ TE $60 \mathrm{~ms}$ ) MR image at the same level as in Fig. 4, again shows a mass in the area of each Achilles tendon 


\section{Diagnosis: Cerebrotendinous xanthomatosis}

The differential diagnosis includes familial and nonfamilial hypercholesterolemic and normocholesterolemic xanthomatosis.

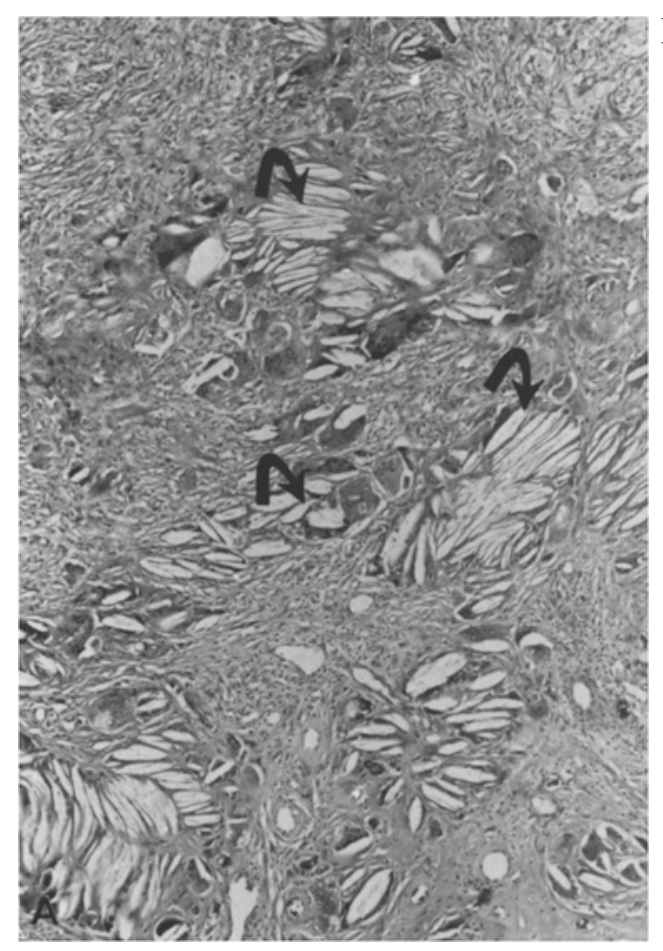

\section{Pathological studies}

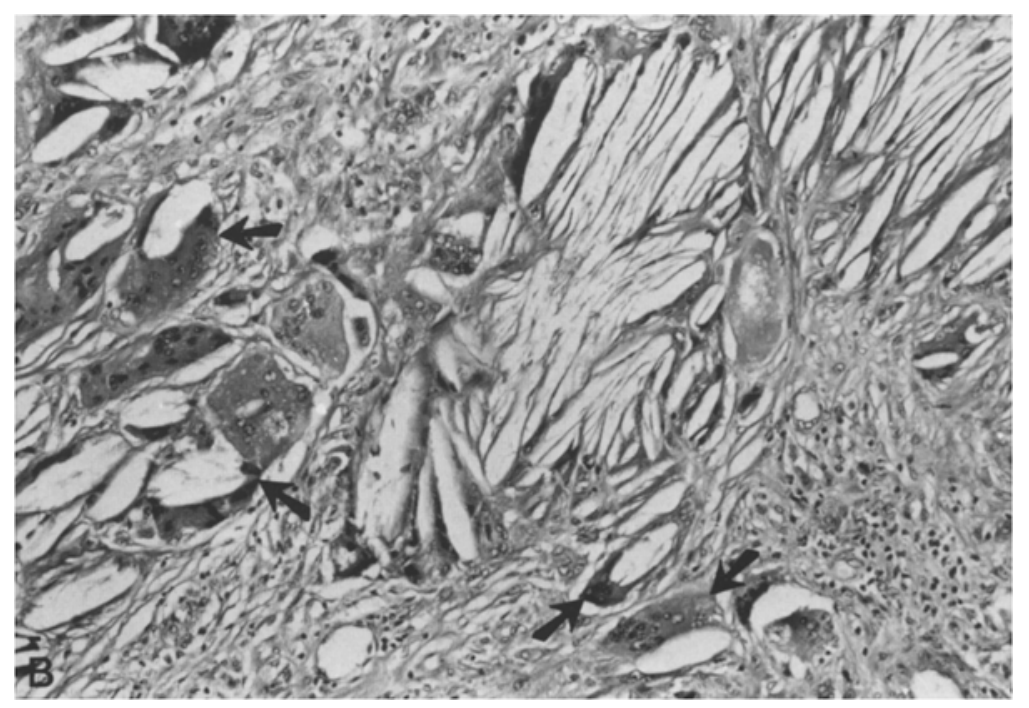

Fig. 6. A A low-power view of an involved tendon demonstrates numerous cleft-like spaces typical of deposits of cholesterol (curved arrows). In addition observe the multinucleated foreign body giant cell inflammatory response associated with the deposition of cholesterol (HE stain $\times 120)$. B A high-power view of an involved tendon demonstrates the numerous nuclei of the multinucleated giant cells (straight arrows) surrounding the many cholesterol clefts $($ HE stain $\times 220)$

\section{Discussion}

Cerebrotendinous xanthomatosis is a rare, autosomal, recessive disease characterized by deposition of cholestanol and cholesterol in the brain, lungs and tendons, associated with a normal or low level of serum cholesterol. The accumulation of these bile acid precursors is said to be secondary to the deficiency of the enzyme mitochondrial C27-steroid 26-hydroxylase, essential for the normal degradation of the cholesterol side chain [1,2]. Clinical features may include progressive cerebellar ataxia and dementia, cataracts, low intelligence, enlarged tendons, myocardial, pulmonary, and endocrine dysfunction, and premature atherosclerosis with normal serum cholesterol. Treatment with chenodeoxycholic acid has resulted in reversal of dementia, improvement or disappearance of pyramidal and cerebellar signs and return of cholestanol levels to norma1 $[3,4]$.

Radiographic abnormalities can be demon- strated on occasion within the central nervous system. Diffusely decreased attenuation of white matter on computed tomography (CT) may be present. Following successful treatment, these CT abnormalities have been shown to be reversible [5]. The findings on MR of a high intensity signal, suggestive of white matter demyelination, have been reported [6]. Our patient had a normal MR and a normal CT examination of the brain.

The biopsy of a tendon demonstrated the characteristic findings of cleft-like spaces (cholesterol clefts), partially surrounded by large multinucleated giant cells (Fig. 6A, B). Our patient had massively enlarged Achilles tendons on physical examination, clearly demonstrated on plain films (Fig. 1). The CT images of the lower extremities showed that the enlarged tendons were of minimally inhomogeneous soft tissue attenuation, without calcification (Fig. 2). T1 and T2-weighted MR 
images demonstrated curvilinear, low intensity structures within the mass of the tendons (Figs. 35). These are believed to represent the normal fibrous tissue of the tendons, inasmuch as they are low in intensity on both $\mathrm{T} 1$ and T2 weighted images. The remaining substance within the affected tendon represents cholesterol and cholestanol in a solid crystalline form, which is responsible for the relatively low intensity signal on the MR images. This observation agrees with the histopathologic examination, which demonstrated classic cholesterol deposition interspersed in the dense fibrous connective tissue of the involved tendon.

Pastershank and colleagues described three affected sisters with massively enlarged Achilles tendons on plain radiographs, strikingly similar in appearance to this case [7]. Previous cases of xanthomas of tendons have been reported in patients with normocholesterolemia or hypercholesterolemia [8-11]. The appearance of such involved tendons might be expected to appear similar to the tendons in patients with cerebrotendinous xanthomatosis, when evaluated by plain films, CT, or MRI imaging.

In summary, the case is presented of a 42-yearold man with cerebrotendinous xanthomatosis, demonstrating markedly enlarged Achilles tendons both on plain radiographs and CT. MR showed that the relatively low intensity signal in the affected tendons correlates with the histopathologic finding of deposition of solid cholesterol crystals interspersed between the dense fibrous connective tissue of tendons.
Acknowledgement. We thank Dr. Husin, Department of Pathology, Sinai Hospital, Detroit, Michigan, for obtaining the histopathologic specimen

\section{References}

1. Skrade S, Birkhen I (1985) A novel route for the biosynthesis of cholestanol, and its significance for the pathogenisis of cerebrotendinous xanthomatosis. Scand J Clin Lab Invest [Suppl] 177:15-21

2. Salen G, Shefer S, Tint GS, Nicolau G, Dayal B, Batta AK (1985) Biosynthesis of bile acids in cerebrotendinous xanthomatosis. Relationship of bile acid pool sizes and synthesis ratio to hydroxylations at $\mathrm{C}-12, \mathrm{C}-25$, and $\mathrm{C}-26$. J Clin Invest 76:744-751

3. Koopman BJ, Wolthers BG, Van-der-malen JC, Waterreus RJ (1985) Bile acid therapies applied to patients suffering from cerebrotendinous xanthomatosis. Clin Chim Acta 152:115-122

4. Pedley TA, Emerson RG, Warner CL, Ronald LP, Salen $G$ (1985) Treatment of cerebrotendinous xanthomatosis with chenodeoxycholic acid. Ann Neurol 18:517-518

5. Berginer VM, Salen G, Shefer S (1984) Long-term treatment of cerebrotendinous xanthomatosis with chenodeoxycholic acid. N Engl J Med 311:1649-1652

6. Swanson PD, Cromwell LD (1986) Magnetic resonance imaging in cerbrotendinous xanthomatosis. Neurology $36: 124-126$

7. Pastershank SP, Yip S, Sodhi HS (1974) Cerebrotendinous xanthomatosis. J Can Assoc Radiol 25:282-286

8. Hughes JD, Meriwether TW III (1971) Familial pseudohypertrophy of tendon Achilles with multisystem disease. South Med J 64:311

9. March HC, Gilbert PD, Kain TM (1957) Hypercholesteremic xanthoma of the tendons. AJR 77:109

10. Weintrob L, Truswell AS (1971) A case of type III hyperlipo-proteinaemia with xanthomata. Br J Radiol 44:215

11. Blomquist G (1962) Xanthoma of the tendon Achilles. Acta Radiol 57:45 